\title{
Participatory Public Media Arts for Social Creativity
}

\author{
Jun $\mathrm{Hu}^{1}$, Feng Wang ${ }^{1,2}$, Mathias Funk ${ }^{1}$, Joep Frens ${ }^{1}$, Yu Zhang ${ }^{1}$, Thom van Boheemen ${ }^{1}$ \\ Chenxi Zhang ${ }^{2}$, Qi Yuan ${ }^{2}$, Hongrui $\mathrm{Qu}^{2}$, Matthias Rauterberg ${ }^{1}$ \\ ${ }^{1}$ Department of Industrial Design, Eindhoven University of Technology, The Netherlands \\ ${ }^{2}$ School of Digital Media, Jiangnan University, China
}

\begin{abstract}
New technologies and materials bring new opportunities to the forms of public arts. In this paper we look back to the three generations of public art forms, from traditional ones with static forms to newer ones with dynamic and then, interactive forms. We look forward to the fourth generation - participatory media arts, for which the artists and designers do not create the public media arts as a final result, but create them as platforms and growing systems for the public to participate and for social creativity to contribute to the artifact - the creation process, together with the results of this process, forms the dynamic media artifact that grows over time. The fourth generation is illustrated with an example of public media art installation designed for the Science and Education New Town, Taicang, China.
\end{abstract}

Keywords - public media arts, social creativity, dynamic forms, interactive forms, participatory forms, growing systems

\section{INTRODUCTION}

The advances in science and technology have been always reflected in art, as it reflects and comments on the technological progress. In the last decades public arts shifted from traditional forms such as stone, canvas and pottery towards new media types. The current development in digital media arts involves a significant amount of new carriers in not only new materials, but also in perusing technology, resulting in new dynamic and interactive forms that require the artists to construct their work from a system view and with a good understanding of human-system interaction and related interface technologies. It is no longer about carving stones and casting bronze; instead it is time to sculpture the interactive experience as a growing system [1].

Looking into the development of public arts, especially the introduction of interactivity, based on the work of Edmonds, Turner and Candy [2], Wang, $\mathrm{Hu}$ and Rauterberg defined three generations of art and generative technology according to the carrying material, technology and interactivity [1]: 1) Static forms: there is no interaction between the art artifact and the viewer, and the artifact does not respond to its context and environment. 2) Dynamic forms: the art artifact has its internal mechanism to change its forms, depending on time or limited to reacting to the changes in its environment such as temperature, sound or light. The viewer is however a passive observer and has no influence on the behavior of the artifact. 3) Interactive forms: the viewer has an active role in influencing the dynamic form of the art object. The input from the viewer can be gesture, motion, sound as well as other human activity that can be captured by the artifact's sensorial layer. When interactivity is introduced, the "dialog" between the viewer and the perceived dynamic form of the artifact can always vary depending on the difficult-to-predict behavior of the human viewer.

In this paper we introduce the fourth generation of the public art forms - participatory forms - Interactive art platforms that allow social interactivity and creativity contribute to the physical and digital parts of the artifact. Artists and designers do not create the public media arts as a final (interactive) result, but create them as platforms for other artists and the public to contribute to the artifact. The creation process, together with the results of this process, forms the dynamic media artifact that grows with the creative input from the social environment and over time. The art piece now become an almost living form that changes its shape reacting on and adapting to the social environment it is embedded in.

\section{Participatory Public Media Arts}

Growing form per se is not a new concept. It is a common practice in garden and landscape design. Sculptures may include living plants or other living materials as part of the creation, or even as the basis for the entire artifact. An example for using tissue engineering is, the Tissue Culture \& Art Project set up in 1996 to explore the possibilities of manipulating living tissues to create and grow semi-living objects and sculptures [3]. Fig. 1(a) depicts the epidermal and connective tissue grown over a glass figurine in a shape of a bomb (by Catts and Zurr [3]). However in most of the cases the growing process is not created or designed for the viewers to interact with or to contribute to. The viewer remains passive, while the artist operates under a closed world assumption creating the art piece as a closed isolated system.

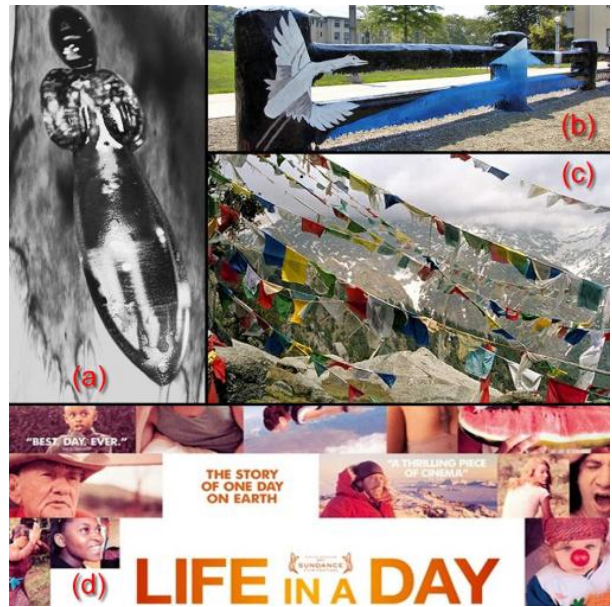

Fig. 1. Growing and participatory arts (images taken from Internet)

Participatory art forms are not new either. "The fence" at Carnegie Mellon (Fig. 1(b)) was set up as a meeting point but later was painted over and over again by the students for advertising or making a statement. In this case the fence is the art platform and the participation of the students makes the fence an interesting piece of art. The contribution of the 
students is part of the physical form - color by layers of paint. Another example is the Tibetian flags that the prayers bring to the temples in the area of Himalaya (Fig. 1(c)). The prepared strings around the temples are created for the participation of the prayers. The process of hanging the flags is a religious practice but the result of it is a breathtaking piece of art, of which the contribution of the prayers is again a physical part.

Participatory media forms are mostly seen in online applications, particularly those that allow a high degree of usercontributed content [4] - examples are blogs, forums, social photo and video services. One of the most recent examples is the movie "Life in a Day" (Fig. 1(d)), directed by Kevin McDonald, generated entirely from amateur video clips of people's lives that were contributed by YouTube users [5]. In this case YouTube is the art platform and the contribution from the users is digital. The involvement of the artist (Kevin McDonald) is at a later stage of production and the result of the social creation is frozen into a final from afterwards.

Participatory public media art form emerges from the above art forms and from the technology development. It integrates the physical and digital forms and content, requires the artist to take into account the future inclusion of the contributions from the public, and opens the possibility to allow social creativity as part of the creation process, aiming at an artifact (in many cases, a system) that grows with the contributions and along with time. What the artist creates is not an artifact in its final form, but a platform for social creativity. The participation process is an interactive process, although the interaction is not necessarily real-time at the same spot, but can be over time and over distance, thanks to the new technologies of digital media and connectivity. The interesting new aspect of such art is that the artist's actions (selection, compilation, refinement, narration) happen over time, automatically and guided by the affordances of the art form. This is why HCI and related disciplines matter so much in this context: the artist needs to predict user actions and creativity in a way and predictively channeling future input into an artistic vision. Next we show an example.

\section{EXAMPLE: PUBliC ART INATALLATION FOR TAICANG}

Taicang is a city near Shanghai. It is a natural sea port. It became the number one port of the world in Ming dynasty, where Zheng He set off his voyages to explore the world [6]. Taicang's cultural heritage is deeply rooted in being a sea port to the world ever since. Only recently Taicang decided to exploit this past importance and fame as one of the development triggers to a new culture and creative industry in the region. One of the ongoing projects is to build a new living and leisure area at the spot of the original shipping embankment (Fig. 2(a)). A public media art installation is designed and will be located at the center square of the area (Fig. 2(b)).

The installation is roughly 10 by 10 meters on its base and 8 meters high. The base features wall reliefs and public displays, and inside it is going to be used as an exhibition space. On top of the base are constructions that give the impressions of a large sail, and the moon rising from the waves. On the surface of the sail are reliefs of Taicang's sea culture. The inner space of the "moon" is also used for mounting projectors. Images, animations and videos can be projected onto the inner surface of sail in the evenings. Projection mapping and edge blending techniques will be used to correct the projection results on the curved surface with reliefs on it. Together with the sound systems installed nearby, it offers a platform for presenting digital media, and for public participation.

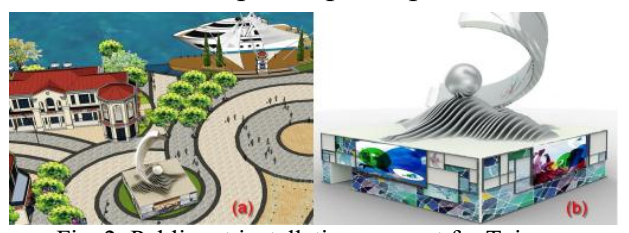

Fig. 2. Public art installation concept for Taicang

One of the concepts to utilize this platform is to allow the public to contribute their photos from social media, for an interactive photo show (Fig. 3), to induce the feeling of social connectedness [7], and to reinstate the historical values of Taicang as port to the world, the place where Zheng He left to explore the world and bring back treasures and cultural influences from overseas. People from Taicang, who live and work overseas, will be able to send back photos and show to the people of Taicang what they are doing and seeing elsewhere in the world,. Local viewers are able to browse through the photos by turning a steering wheel, and one of the photos will appear in large scale in the sail. The amount of the time of the photo being exposed will send back to the contributor of the photo as feedback. The concept has been prototyped on scale models, as shown in Fig. 3.

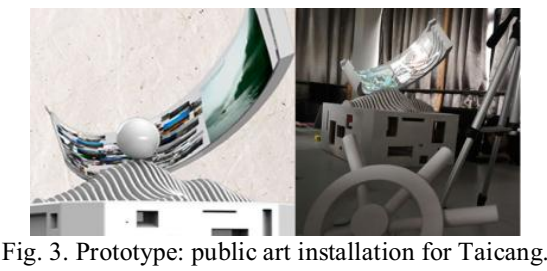

\section{CONCLUDING REMARKS}

Based on our previous work, we introduced the fourth generation of the public art forms - participatory forms. The public media art installation for the Taicang city is used as an example. The installation is designed with public displays and projections that allow the public to contribute digital content to the art and the art will be an integrated entity of the creativity of the artists, and the social creativity from the public.

\section{REFERENCES}

1. Wang, F., J. Hu, and M. Rauterberg, New Carriers, Media and Forms of Public Digital Arts, in Culture and Computing 2012, Springer-Verlag Berlin Heidelberg: Hangzhou, China. p. 83-93.

2. Edmonds, E., G. Turner, and L. Candy, Approaches to interactive art systems, in Proceedings of the 2nd international conference on Computer graphics and interactive techniques in Australasia and South East Asia2004, ACM: Singapore. p. 113-117.

3. Catts, O. and I. Zurr, Growing semi-living sculptures: The tissue culture \& art project. Leonardo, 2002. 35(4): p. 365-370.

4. Cameron, D., Mashup: digital media and drama conventions. Drama education with digital technology, 2009: p. 52-66.

5. Hoffman, J., Film: Science shines at Sundance. Nature, 2011. 469(7330): p. 298-298.

6. Levathes, L., When China ruled the seas. 1994: Oxford University Press.

7. Le, D., M. Funk, and J. Hu, Blobulous: Computers As Social Actors., in CHI 2013 workshop: Experiencing Interactivity in Public Spaces (EIPS)2013: Paris. 


\section{Proceedings}

\section{International Conference on Culture and Computing Culture and Computing 2013}

16-18 September 2013

Kyoto, Japan 


\section{Message from the Chairs Culture and Computing 2013}

Welcome to 2013 International Conference on Culture and Computing (Culture and Computing 2013).

International communities are facing various problems in different topic areas such as: population demographic shifts, energy use and creation, the environment, and food supply. It is necessary to build a global consensus for resolving problems within these topic areas. Unfortunately, there are difficulties that hinder communication among cultures. It is imperative to develop information and communication technologies that encourage mutual understanding and bridge the difference in cultures.

Several research directions impinge on the relations between culture and computing: archiving cultural heritage via ICT (cf. digital archives), empowering humanities researches via ICT (cf. digital humanities), creating art and expressions via ICT (cf. media art), generating culturally-directed behavior (cf. cultural agent), supporting multi-language, multi-cultural societies via ICT (cf. intercultural collaboration), and understanding new cultures born in the Internet and the Web (cf. net culture).

This year, Culture and Computing is back to Kyoto, the cultural heart of Japan, to provide an opportunity to share research issues and discuss the future of culture and computing. The conference is organized so as to exhibit the integration of state-of-the-art cultural computing technologies and Japanese traditional culture, along with several co-located events.

We have a single session Main Track and two Special Track sessions. The Main Track will present a collection of scientific or engineering research results that include, archiving cultural heritages, information environments for humanity studies, art and design, intercultural communication and collaboration, culturally situated agents and simulations, and analysis of new cultures in the Internet and Web.

The Special Tracks are collections of short papers, and are organized for the purpose of encouraging discussions in hot areas. This year, we have Special Tracks for "Digital Humanities" and "Culture based Media Art \& Music." The Digital Humanities captures the trend in research and education in the Humanities that has emerged over the last decade. While researchers have not reached consensus on its definition, digital data available online all over the world as well as the global collaboration through the web will usher in a paradigm shift in Humanities research. Culture based Media Art \& Music is anticipated in the new century. It has been said that intelligence, sensitivity, and consciousness are the central and most important parts of humanity. The 21st century can be an era of sensitivity if it adopts the essential basis of Asian culture. The generality, value, and importance of culture should be emphasized to the world.

The conference proceedings include both full papers presented in the Main Track, together with short papers discussed in poster sessions and Special Tracks. We are sure you will find your participation in the conference fruitful and hope that it is enjoyable. We are grateful to the authors, presenters, and delegates for their contributions. We would also like to express our special thanks to our program committee members and all the external reviewers for reviewing all the papers. Finally, we wish to thank the IEEE Computer Society Conference Publishing Services for their support in compiling the proceedings.

Kozaburo Hachimura, Toru Ishida and Naoko Tosa

Culture and Computing 2013 General Co-Chairs

Donghui Lin and Akira Maeda

Culture and Computing 2013 Program Co-Chairs 


\title{
IEEE Computer Society Technical \& Conference Activities Board
}

\author{
T\&C Board Vice President \\ Paul R. Croll \\ Computer Sciences Corporation
}

IEEE Computer Society Staff

Evan Butterfield, Director of Products and Services

Lynne Harris, CMP, Senior Manager, Conference Support Services

Alicia Stickley, Senior Manager, Publishing Operations

Silvia Ceballos, Manager, Conference Publishing Services

Patrick Kellenberger, Supervisor, Conference Publishing Services

\section{IEEE Computer Society Publications}

The world-renowned IEEE Computer Society publishes, promotes, and distributes a wide variety of authoritative computer science and engineering texts. These books are available from most retail outlets. Visit the CS Store at http://www.computer.org/portal/site/store/index.jsp for a list of products.

\section{IEEE Computer Society Conference Publishing Services (CPS)}

The IEEE Computer Society produces conference publications for more than 300 acclaimed international conferences each year in a variety of formats, including books, CD-ROMs, USB Drives, and on-line publications. For information about the IEEE Computer Society's Conference Publishing Services (CPS), please e-mail: cps@computer.org or telephone+1-714-821-8380. Fax +1-714-761-1784. Additional information about Conference Publishing Services (CPS) can be accessed from our web site at: http://www.computer.org/cps

Revised: 18 January 2012

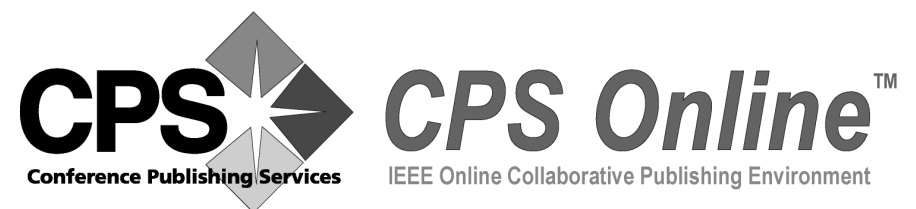

CPS Online is our innovative online collaborative conference publishing system designed to speed the delivery of price quotations and provide conferences with real-time access to all of a project's publication materials during production, including the final papers. The CPS Online workspace gives a conference the opportunity to upload files through any Web browser, check status and scheduling on their project, make changes to the Table of Contents and Front Matter, approve editorial changes and proofs, and communicate with their CPS editor through discussion forums, chat tools, commenting tools and e-mail.

The following is the URL link to the CPS Online Publishing Inquiry Form: http://www.computer.org/portal/web/cscps/quote 